Orthopäde $2019 \cdot 48: 1057$

https://doi.org/10.1007/s00132-019-03835-1

Online publiziert: 14 . November 2019

(c) Springer Medizin Verlag $\mathrm{GmbH}$, ein Teil von Springer Nature 2019

\author{
J. Friesenbichler $\cdot$ M. Bergovec $\cdot$ W. Maurer-Ertl $\cdot$ P. Reinbacher $\cdot$ M. Maier $\cdot$ \\ F. Amerstorfer $\cdot$ A. Leithner \\ Univ. Klinik für Orthopädie und Traumatologie, Medizinische Universität Graz, Graz, Österreich
}

\title{
Erratum zu: Silberbeschichtung auf Tumorendoprothesen
}

\section{Erratum zu:}

Orthopäde 2019

https://doi.org/10.1007/s00132-01903720-x

Sehr geehrte Leserin, sehr geehrter Leser,

im Beitrag „Silberbeschichtung auf Tumorendoprothesen" in Der Orthopäde 07/2019 ist es im Abschnitt Messung der Silberionen im Blut zu einer fehlerhaften Mengenangabe gekommen. Korrekt muss es lauten: Scoccianti et al. [18] berichteten 2016 von den Silberwerten im Blut bei 33 Patienten mit dem modifizierten Megasystem-C (Waldemar Link, Hamburg, Deutschland) und der PorAg-Silberoberflächenbeschichtung. Die mittleren Silberspiegel im Blut lagen zwischen 0,41 und 5,33 ppb 24 und 36 Monaten nach der Operation und zwischen 0,28 und 0,86 ppb im Urin. Im Vergleich zu den Messwerten einer vorher publizierten Serie, bei welcher ein anderer Implantattyp (MUTARS, Implantcast, Buxtehude, Deutschland) verwendet wurde, waren diese gemessenen Werte im vergleichbaren Bereich bzw. sogar tendenziell niedriger [11]. Auch in dieser Studie wurden weder klinische Anzeichen einer Argyrie, noch lokale oder systemische Nebenwirkungen im Zusammenhang mit den Silberkonzentrationen festgestellt [19].

Wir bitten, die korrekten Werte zu beachten, und entschuldigen uns für den Fehler.

\author{
Korrespondenzadresse \\ PD Dr. J. Friesenbichler \\ Univ. Klinik für Orthopädie und Traumatologie, \\ Medizinische Universität Graz \\ Auenbruggerplatz 5, $8036 \mathrm{Graz}$, Österreich \\ joerg.friesenbichler@medunigraz.at
}

\section{Literatur}

11. Hardes J, Ahrens H, Gebert C et al (2007) Lack of toxicological side-effects in silver-coated megaprostheses in humans. Biomaterials 28:2869-2875

18. Scoccianti G, Frenos F, Beltram G et al (2016) Levels of silver ions in body fluids and clinical results in silver-coated megaprostheses after tumour, trauma or failed arthroplasty. Injury 47(Suppl 4):S11-S16

19. Shivaram A, Bose S, Bandyopadhyay A (2017) Understanding long-term silver release from surface modified porous titanium implants. Acta Biomater 58:550-560 